Homology, Homotopy and Applications, vol.13(1), 2011, pp.121-142

\title{
THE BROWN-GOLASIŃSKI MODEL STRUCTURE ON STRICT $\infty$-GROUPOIDS REVISITED
}

\author{
DIMITRI ARA AND FRANÇOIS MÉTAYER
}

\author{
(communicated by Ronald Brown)
}

\begin{abstract}
We prove that the folk model structure on strict $\infty$-categories transfers to the category of strict $\infty$-groupoids (and more generally to the category of strict $(\infty, n)$-categories), and that the resulting model structure on strict $\infty$-groupoids coincides with the one defined by Brown and Golasiński via crossed complexes.
\end{abstract}

\section{Introduction}

In [4], Brown and Golasiński build a model structure on the category of crossed complexes. In an earlier work [5], Brown and Higgins established an equivalence of categories between crossed complexes and strict $\infty$-groupoids, whence a model structure on the latter category.

On the other hand, there is a "folk" model structure on strict $\infty$-categories recently discovered by Lafont, Worytkiewicz and the second author [10], which extends previously known model structures on categories $[\mathbf{8}]$ and 2-categories [9]. Note that this model structure is also known as the "natural" or the "categorical" model structure by various authors.

This immediately raised the questions whether the folk model structure on $\infty$-categories may be transferred to $\infty$-groupoids by inclusion, and in that case whether the Brown-Golasiński model structure may be recovered this way.

The purpose of the present work is to show that both questions have affirmative answers.

Our paper is organized as follows: In Section 1, we recall the basic definitions of strict $\infty$-categories and strict $\infty$-groupoids. Then, in Section 2, we describe the Brown-Golasiński model structure on crossed complexes and $\infty$-groupoids. In Section 3, we prove the transfer theorem leading to the definition of the folk model structure on strict $\infty$-groupoids. Our proof uses neither crossed complexes nor the existence of the Brown-Golasiński model structure. Moreover, it also applies to the category of strict $(\infty, n)$-categories for a fixed $n$ (that is $\infty$-categories such that every $m$-arrow is invertible for $m>n$ ). Finally, in Section 4, we show that the two model structures previously defined on $\infty$-groupoids are in fact the same.

Received October 14, 2010, revised January 18, 2011; published on May 16, 2011. 2000 Mathematics Subject Classification: 18D05, 18G55, 55U35.

Key words and phrases: $\infty$-category, $\infty$-groupoid, crossed complex, model category. Article available at http://intlpress.com/HHA/v13/n1/a5 and doi:10.4310/HHA.2011.v13.n1.a5 Copyright (C) 2011, Dimitri Ara and François Métayer. Permission to copy for private use granted. 


\section{Acknowledgements}

We thank Ronald Brown and Urs Schreiber, whose questions motivated us to go further in this work.

\section{Strict $\infty$-groupoids}

The purpose of this section is to introduce the definitions and notations about strict $\infty$-groupoids and their weak equivalences that we will use in the sequel of this paper. Our presentation is essentially the same as the one given in [2].

\subsection{Globular sets}

Let us denote by $\mathbf{O}$ the globular category, that is, the category generated by the graph

$$
0 \underset{\tau_{0}}{\stackrel{\sigma_{0}}{\longrightarrow}} 1 \underset{\tau_{1}}{\stackrel{\sigma_{1}}{\longrightarrow}} \cdots \underset{\tau_{i-1}}{\stackrel{\sigma_{i-1}}{\longrightarrow}} i \underset{\tau_{i}}{\stackrel{\sigma_{i}}{\longrightarrow}} i+1 \underset{\tau_{i+1}}{\stackrel{\sigma_{i+1}}{\longrightarrow}} \cdots
$$

under the coglobular relations

$$
\sigma_{i+1} \sigma_{i}=\tau_{i+1} \sigma_{i} \quad \text { and } \quad \sigma_{i+1} \tau_{i}=\tau_{i+1} \tau_{i}, \quad i \geqslant 0
$$

A globular set or $\infty$-graph is a presheaf on $\mathbf{O}$. A globular set $X$ amounts to a diagram of sets

$$
\cdots \underset{t_{n+1}}{\stackrel{s_{n+1}}{\longrightarrow}} X_{n+1} \underset{t_{n}}{\stackrel{s_{n}}{\longrightarrow}} X_{n} \underset{t_{n-1}}{\stackrel{s_{n-1}}{\longrightarrow}} \cdots \underset{t_{1}}{\stackrel{s_{1}}{\longrightarrow}} X_{1} \underset{t_{0}}{\stackrel{s_{0}}{\longrightarrow}} X_{0}
$$

satisfying the globular relations

$$
s_{i} s_{i+1}=s_{i} t_{i+1} \quad \text { and } t_{i} s_{i+1}=t_{i} t_{i+1}, \quad i \geqslant 0 .
$$

For $i \geqslant j \geqslant 0$, we will denote by $s_{j}^{i}$ and $t_{j}^{i}$ the maps from $X_{i}$ to $X_{j}$ defined by

$$
s_{j}^{i}=s_{j} \cdots s_{i-2} s_{i-1} \quad \text { and } \quad t_{j}^{i}=t_{j} \cdots t_{i-2} t_{i-1} .
$$

If $X$ is a globular set, we will call $X_{0}$ the set of objects of $X$ and $X_{n}$ for $n \geqslant 0$ the set of $n$-arrows or $n$-cells. The notation $u: x \rightarrow y$ will mean that $u$ is an $n$-arrow for $n \geqslant 1$ whose source is an $(n-1)$-arrow $x$ (that is $s_{n-1}(u)=x$ ) and whose target is an $(n-1)$-arrow $y$ (that is $t_{n-1}(u)=y$ ). We will say that two $n$-arrows $u$ and $v$ are parallel if either $n=0$, or $n \geqslant 1$ and $u, v$ have same source and same target. For $i \geqslant j \geqslant 0$, if $u$ is an $i$-arrow, we will often write $s_{j}(u)$ for $s_{j}^{i}(u)$ and similarly $t_{j}(u)$ for $t_{j}^{i}(u)$.

If $u$ and $v$ are $n$-arrows, $X(u, v)$ will denote the globular set whose $k$-arrows are the $(n+k+1)$-arrows $a$ of $G$ such that $s_{n}(a)=u$ and $t_{n}(a)=v$. In particular, $X(u, v)_{0}$ is the set of $(n+1)$-arrows $a: u \rightarrow v$ in $X$.

\section{2. $\quad$ Strict $\infty$-categories}

An $\infty$-precategory is a globular set $C$ endowed with maps

$$
\begin{aligned}
& *_{j}^{i}:\left(X_{i}, s_{j}^{i}\right) \times_{X_{j}}\left(t_{j}^{i}, X_{i}\right) \rightarrow X_{i}, \quad i>j \geqslant 0, \\
& k_{i}: X_{i-1} \rightarrow X_{i}, \quad i \geqslant 1,
\end{aligned}
$$


such that

1. For every $(u, v)$ in $\left(X_{i}, s_{j}^{i}\right) \times_{X_{j}}\left(t_{j}^{i}, X_{i}\right)$ with $i>j \geqslant 0$, we have

$$
s_{i-1}\left(u *_{j}^{i} v\right)=\left\{\begin{array}{ll}
s_{i-1}(v), & j=i-1 \\
s_{i-1}(u) *_{j}^{i-1} s_{i-1}(v), & j<i-1
\end{array} ;\right.
$$

2. For every $(u, v)$ in $\left(X_{i}, s_{j}^{i}\right) \times_{X_{j}}\left(t_{j}^{i}, X_{i}\right)$ with $i>j \geqslant 0$, we have

$$
t_{i-1}\left(u *_{j}^{i} v\right)= \begin{cases}t_{i-1}(u), & j=i-1 \\ t_{i-1}(u) *_{j}^{i-1} t_{i-1}(v), & j<i-1\end{cases}
$$

3. For every $u$ in $X_{i}$ with $i \geqslant 0$, we have

$$
s_{i} k_{i+1}(u)=u=t_{i} k_{i+1}(u) .
$$

For $i \geqslant j \geqslant 0$, we will denote by $k_{i}^{j}$ the map from $X_{j} \rightarrow X_{i}$ defined by

$$
k_{i}^{j}=k_{i} \cdots k_{j+2} k_{j+1} .
$$

If $u$ and $v$ are $n$-arrows for $n \geqslant 1$ of an $\infty$-precategory, we will often write $u *_{k} v$ for $u *_{k}^{n} v$. If $u$ is an $n$-arrow, we will often write $1_{u}$ for the iterated identity $k_{m}^{n}(u)$ in a dimension $m \geqslant n$ clear by the context.

Definition 1.1. A strict $\infty$-category is an $\infty$-precategory $X$ such that the following axioms are satisfied:

1. Associativity. For every $(u, v, w)$ in $\left(X_{i}, s_{j}^{i}\right) \times_{X_{j}}\left(t_{j}^{i}, X_{i}, s_{j}^{i}\right) \times_{X_{j}}\left(t_{j}^{i}, X_{i}\right)$ with $i>j \geqslant 0$, we have

$$
\left(u *_{j} v\right) *_{j} w=u *_{j}\left(v *_{j} w\right)
$$

2. Exchange law. For every $\left(u, u^{\prime}, v, v^{\prime}\right)$ in

$$
\left(X_{i}, s_{j}^{i}\right) \times_{X_{j}}\left(t_{j}^{i}, X_{i}, s_{k}^{i}\right) \times_{X_{k}}\left(t_{k}^{i}, X_{i}, s_{j}^{i}\right) \times_{X_{j}}\left(t_{j}^{i}, X_{i}\right),
$$

with $i>j>k \geqslant 0$, we have

$$
\left(u *_{j} u^{\prime}\right) *_{k}\left(v *_{j} v^{\prime}\right)=\left(u *_{k} v\right) *_{j}\left(u^{\prime} *_{k} v^{\prime}\right)
$$

3. Units. For every $u$ in $X_{i}$ with $i \geqslant 1$ and every $j$ such that $i>j \geqslant 0$, we have

$$
u *_{j} 1_{s_{j}(u)}=u=1_{t_{j}(u)} *_{j} u
$$

4. Functoriality of units. For every $(u, v)$ in $\left(X_{i}, s_{j}^{i}\right) \times_{X_{j}}\left(t_{j}^{i}, X_{i}\right)$ with $i>j \geqslant 0$, we have the following equality between $(i+1)$-arrows:

$$
1_{u *_{j} v}=1_{u} *_{j} 1_{v} .
$$

A morphism of strict $\infty$-categories or $\infty$-functor is a morphism of globular sets compatible with the maps $*_{j}^{i}$ and $k_{i}$.

We will denote by $\infty$-Cat the category of $\infty$-categories. This category is a full reflexive subcategory of the presheaf category of globular sets. Moreover, it is stable under filtered colimits. Hence, by Theorem 1.46 of $[\mathbf{1}], \infty$-Cat is locally presentable.

Note that if $u$ and $v$ are two $n$-arrows of a strict $\infty$-category $C$, the globular set $C(u, v)$ inherits a structure of strict $\infty$-category. 


\section{3. $\quad$ Strict $\infty$-groupoids}

Let $C$ be a strict $\infty$-category and $u$ an $i$-arrow for $i \geqslant 1$. For $j$ such that $0 \leqslant j<i$, a $*_{j}^{i}$-inverse $v$ of $u$ is an $i$-arrow such that $s_{j}(v)=t_{j}(u)$ and $t_{j}(v)=s_{j}(u)$, satisfying

$$
u *_{j} v=1_{t_{j}(u)} \text { and } \quad v *_{j} u=1_{s_{j}(u)} .
$$

It is easy to see that if it exists, such an inverse is unique. For $i>j \geqslant 0$, we will say that $C$ admits $*_{j}^{i}$-inverses if every $i$-arrow of $C$ admits a $*_{j}^{i}$-inverse.

Definition 1.2. A strict $\infty$-groupoid is a strict $\infty$-category which admits $*_{j}^{i}$-inverses for every $i>j \geqslant 0$. We will denote by $\infty$-Grp the full subcategory of $\infty$-Cat whose objects are strict $\infty$-groupoids.

Let $n \geqslant 0$. A strict $(\infty, n)$-category is a strict $\infty$-category which admits $*_{j}^{i}$-inverses for every $i>j \geqslant n$. We will denote by $(\infty, n)$-Cat the full subcategory of $\infty$-Cat whose objects are strict $(\infty, n)$-categories. Note that for $n=0$ we recover the category of strict $\infty$-groupoids.

The same argument as for $\infty$-Cat shows that $\infty$-Grp is a locally presentable category.

If $G$ is a strict $\infty$-groupoid and $u$ is an $i$-arrow of $G$ for $i \geqslant 1$, we will denote by $w_{j}^{i}(u)$ or simply by $w_{j}(u)$ the $*_{j}^{i}$-inverse of $u$ and by $u^{-1}$ the $*_{i-1}^{i}$-inverse. Note that if $u$ and $v$ are two $n$-arrows of a strict $\infty$-groupoid $G$, the strict $\infty$-category $G(u, v)$ is a strict $\infty$-groupoid.

Proposition 1.3. Let $C$ be a strict $\infty$-category. Then the following assertions are equivalent:

1. $C$ is a strict $\infty$-groupoid;

2. $C$ admits $*_{i-1}^{i}$-inverses for every $i \geqslant 1$;

3. $C$ admits $*_{0}^{i}$-inverses for every $i \geqslant 1$;

4. For all $i \geqslant 1$, there exists $j$ satisfying $0 \leqslant j<i$ such that $C$ admits $*_{j}^{i}$-inverses.

Proof. By induction, it suffices to show that for every $i>j>k \geqslant 0$, if $C$ admits $*_{k}^{j}$-inverses, then $C$ admits $*_{k}^{i}$-inverses if and only if it admits $*_{j}^{i}$-inverses. By using the fact that the 2-graph

$$
C_{i} \underset{t_{j}^{i}}{\stackrel{s_{j}^{i}}{\longrightarrow}} C_{j} \underset{t_{k}^{j}}{\stackrel{s_{k}^{j}}{\longrightarrow}} C_{k}
$$

has a natural structure of 2-category, one can assume that $k=0, j=1$ and $i=2$. The result is thus a consequence of the following lemma.

Lemma 1.4. Let $C$ be a 2-category whose 1-arrows are invertible. Then a 2-arrow is invertible for horizontal composition (i.e., $*_{0}^{2}$ ) if and only if it is invertible for vertical composition (i.e., $*_{1}^{2}$ ).

Proof. Let $a: u \rightarrow v$ be a 2-arrow. Suppose $a$ admits a horizontal inverse $a^{*}$. Then $v *_{0} a^{*} *_{0} u$ is a vertical inverse. Conversely, suppose $a$ admits a vertical inverse $a^{-1}$. Then $v^{-1} *_{0} a^{-1} *_{0} u^{-1}$ is a horizontal inverse. 


\subsection{Weak equivalences of strict $\infty$-groupoids}

Let $G$ be a strict $\infty$-groupoid. An $n$-arrow $u$ of $G$ is homotopic to another $n$-arrow $v$ if there exists an $(n+1)$-arrow from $u$ to $v$. This implies that the arrows $u$ and $v$ are parallel. If $u$ is homotopic to $v$, we will write $u \sim v$. The relation $\sim$ is an equivalence relation on $G_{n}$ : the properties with respect to source and target of the maps $k_{n+1}$, $w_{n-1}^{n}$ and $*_{n-1}^{n}$ imply respectively that $\sim$ is reflexive, symmetric and transitive.

Let us denote by $\overline{G_{n}}$ the quotient of $G_{n}$ by $\sim$. The composition

$$
*_{n-1}^{n}: G_{n} \times_{G_{n-1}} G_{n} \rightarrow G_{n}
$$

induces a map

$$
*_{n-1}^{n}: \overline{G_{n}} \times_{G_{n-1}} \overline{G_{n}} \rightarrow \overline{G_{n}},
$$

thanks to the properties with respect to source and target of the composition $*_{n-1}^{n+1}$. For $n \geqslant 1$, we can thus define a groupoid $\varpi_{n}(G)$ whose objects are $(n-1)$-arrows of $G$ and whose morphisms are elements of $\overline{G_{n}}$. It is clear that $\varpi_{n}$ defines a functor from the category of strict $\infty$-groupoids to the category of groupoids.

Definition 1.5. The set of connected components of $G$ is

$$
\pi_{0}(G)=\pi_{0}\left(\varpi_{1}(G)\right)=\overline{G_{0}} .
$$

For $n \geqslant 1$ and $x$ an object of $G$, the $n$-th homotopy group of $G$ at $x$ is

$$
\pi_{n}(G, x)=\pi_{1}\left(\varpi_{n}(G), 1_{x}\right)=\operatorname{Aut}_{\varpi_{n}(G)}\left(1_{x}\right) .
$$

By functoriality of the $\varpi_{n}$ 's, $\pi_{0}$ induces a functor from the category of strict $\infty$-groupoids to the category of sets, and $\pi_{n}$, for $n \geqslant 1$, induces a functor from the category of pointed strict $\infty$-groupoids to the category of groups. By the EckmannHilton argument, the groups $\pi_{n}(G, x)$ are abelian for $n \geqslant 2$. More generally, if $u$ and $v$ are two $(n-1)$-arrows for $n \geqslant 1$ we set

$$
\pi_{n}(G, u, v)=\operatorname{Hom}_{\varpi_{n}(G)}(u, v) \quad \text { and } \quad \pi_{n}(G, u)=\pi_{n}(G, u, u) .
$$

Definition 1.6. A morphism $f: G \rightarrow H$ of strict $\infty$-groupoids is a weak equivalence of strict $\infty$-groupoids if the map $\pi_{0}(f): \pi_{0}(G) \rightarrow \pi_{0}(H)$ is a bijection and if, for all $n \geqslant 1$ and all object $x$ of $G$, the morphism $\pi_{n}(f, x): \pi_{n}(G, x) \rightarrow \pi_{n}(H, f(x))$ is a group isomorphism. We will denote by $\mathcal{W}_{\mathrm{gr}}$ the class of such weak equivalences.

Proposition 1.7. Let $f: G \rightarrow H$ be a morphism of strict $\infty$-groupoids. The following conditions are equivalent:

1. $f$ is a weak equivalence of strict $\infty$-groupoids;

2. $\pi_{0}(f): \pi_{0}(G) \rightarrow \pi_{0}(H)$ is a bijection, and for all $n \geqslant 1$ and every $(n-1)$-arrow $u$ of $G, f$ induces a bijection

$$
\pi_{n}(G, u) \rightarrow \pi_{n}(H, f(u))
$$

3. $\varpi_{1}(f): \varpi_{1}(G) \rightarrow \varpi_{1}(H)$ is an equivalence of categories, and for all $n \geqslant 2$ and every pair $(u, v)$ of parallel $(n-1)$-arrows of $G, f$ induces a bijection

$$
\pi_{n}(G, u, v) \rightarrow \pi_{n}(H, f(u), f(v)) ;
$$


4. $\varpi_{1}(f): \varpi_{1}(G) \rightarrow \varpi_{1}(H)$ is full and essentially surjective, and for all $n \geqslant 2$ and every pair $(u, v)$ of parallel $(n-1)$-arrows of $G, f$ induces a surjection

$$
\pi_{n}(G, u, v) \rightarrow \pi_{n}(H, f(u), f(v)) .
$$

Proof of $1 \Rightarrow 2$. The case $n=1$ is obvious. Let $n \geqslant 2$ and let $u$ be an $(n-1)$-arrow of $G$. Set $x=s_{0}(u)$. The map

$$
\pi_{n}(G, x) \rightarrow \pi_{n}(G, u),
$$

which sends an $n$-arrow $a: 1_{x} \rightarrow 1_{x}$ to the $n$-arrow $1_{u} *_{0} a: u \rightarrow u$, is an isomorphism. Moreover, $f$ commutes with this isomorphism, that is, the square

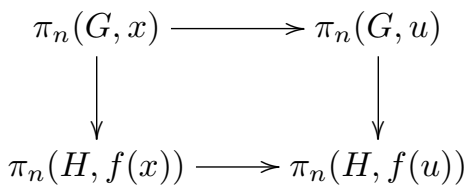

is commutative. The map $\pi_{n}(G, u) \rightarrow \pi_{n}(H, f(u))$ is thus a bijection for $n \geqslant 2$.

Proof of $2 \Rightarrow 3$. Let $n \geqslant 1$ and let $u, v$ be two parallel $(n-1)$-arrows of $G$. Suppose there exists an $n$-arrow $a: u \rightarrow v$ in $G$. The map

$$
\pi_{n}(G, u) \rightarrow \pi_{n}(G, u, v)
$$

which sends an $n$-arrow $b: u \rightarrow u$ to the $n$-arrow $a *_{n-1} b: u \rightarrow v$, is a bijection. Moreover, $f$ commutes with this bijection, that is, the square

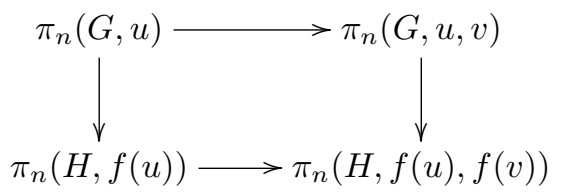

is commutative.

Thus to conclude it suffices to show that if there exists an $n$-arrow $b: f(u) \rightarrow f(v)$ in $H$, then there exists an $n$-arrow $a: u \rightarrow v$ in $G$. It is clear when $n=1$ by injectivity of $\pi_{0}(f)$. Let $n \geqslant 2$ and let $b: f(u) \rightarrow f(v)$ be an $n$-arrow of $H$. Set $x=s_{n-2}(u)$. The arrow $1_{f(u)^{-1} *_{n-2}} b$ is an $n$-arrow of $H$ from $1_{f(x)}: f(x) \rightarrow f(x)$ to $f(u)^{-1} *_{n-2}$ $f(v): f(x) \rightarrow f(x)$. Since the map

$$
\pi_{n-1}(G, x) \rightarrow \pi_{n-1}(H, f(x))
$$

is injective, there exists an $n$-arrow $a^{\prime}$ of $G$ from $1_{x}$ to $u^{-1} *_{n-2} v$. Then $a=1_{u} *_{n-2} a^{\prime}$ is an $n$-arrow of $G$ from $u$ to $v$.

Proof of $3 \Rightarrow 1$. Obvious.

Proof of $4 \Rightarrow 3$. Let $n \geqslant 1$, let $u, v$ be two parallel $(n-1)$-arrows of $G$ and let $a, b$ be two $n$-arrows from $u$ to $v$. Suppose we have $f(a)=f(b)$ in $\pi_{n}(H, f(u), f(v))$. Then there exists an $(n+1)$-arrow of $H$ from $f(a)$ to $f(b)$. By surjectivity of the map

$$
\pi_{n+1}(G, a, b) \rightarrow \pi_{n+1}(H, f(a), f(b)),
$$

there exists an $(n+1)$-arrow in $G$ from $a$ to $b$. Thus $a=b$ in $\pi_{n}(G, u, v)$.

Proof of $3 \Rightarrow 4$. Obvious. 


\section{The Brown-Golasiński model structure}

In [4], Brown and Golasiński introduce a model category structure on the category of crossed complexes. By the equivalence of categories between crossed complexes and strict $\infty$-groupoids constructed in [5], this model structure induces a model structure on strict $\infty$-groupoids. The purpose of this section is to describe this model structure.

\subsection{Crossed complexes}

Let us denote by Grp the category of groups and by $\mathbf{C G r p}_{\geqslant 2}$ the category of homological complexes of (not necessarily commutative) groups in dimension greater or equal to 2 , that is of sequences of morphisms of groups

$$
\cdots \rightarrow C_{n} \stackrel{d_{n}}{\longrightarrow} C_{n-1} \rightarrow \cdots \rightarrow C_{3} \stackrel{d_{3}}{\longrightarrow} C_{2}
$$

such that for every $n \geqslant 4$, we have $d_{n-1} d_{n}=1$, where 1 denotes the unit element of $C_{n-2}$. We have an inclusion functor $i_{2}: \mathbf{G r p} \rightarrow \mathbf{C G r p}_{\geqslant 2}$ which sends a group $G$ to the complex concentrated in degree 2 on $G$.

Let $C_{\leqslant 1}$ be a groupoid. We will denote by $C_{0}$ its set of objects and by $C_{1}(x, y)$ the set of morphisms from an object $x$ to an object $y$ in $C_{\leqslant 1}$. Let $C_{1}: C_{\leqslant 1} \rightarrow \operatorname{Grp}$ be the functor defined in the following way: an object $x$ of $C_{\leqslant 1}$ is sent to the group $C_{1}(x)=C_{1}(x, x)$; a morphism $u: x \rightarrow y$ of $C_{\leqslant 1}$ is sent to the morphism of conjugation by $u$, i.e.,

$$
\begin{aligned}
C_{1}(x) & \rightarrow C_{1}(y) \\
v & \mapsto u v u^{-1} .
\end{aligned}
$$

Definition 2.1. A precrossed complex consists of

- A groupoid $C_{\leqslant 1}$;

- A functor $C_{\geqslant 2}: C_{\leqslant 1} \rightarrow \mathbf{C G r p}_{\geqslant 2}$;

- An augmentation of $C_{\geqslant 2}$ over $C_{1}$, that is a natural transformation $d_{2}: C_{\geqslant 2} \rightarrow$ $i_{2} C_{1}$.

Explicitly, a precrossed complex is given by

- For every $x$ in $C_{0}$, a complex

$$
C_{\geqslant 2}(x)=\cdots \rightarrow C_{n}(x) \stackrel{d_{n}}{\longrightarrow} C_{n-1}(x) \rightarrow \cdots \rightarrow C_{3}(x) \stackrel{d_{3}}{\longrightarrow} C_{2}(x) ;
$$

- For every $x$ in $C_{0}$, a morphism $d_{2}: C_{2}(x) \rightarrow C_{1}(x)$;

- For every $n \geqslant 2$ and every morphism $u: x \rightarrow y$ of $C_{\leqslant 1}$, a morphism $C_{n}(x) \rightarrow$ $C_{n}(y)$ of groups functorial in $u$,

such that for every morphism $u: x \rightarrow y$ of $C_{\leqslant 1}$, the diagram

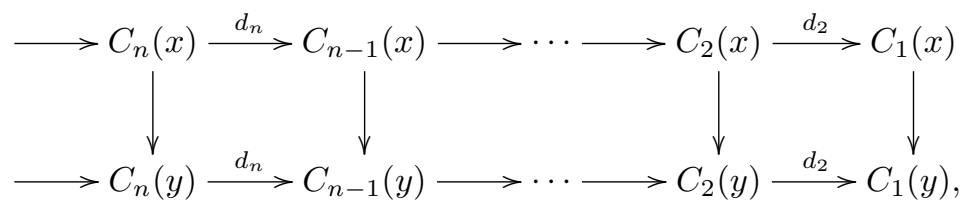

where $C_{1}(x) \rightarrow C_{1}(y)$ is the conjugation by $u$, is commutative. 
If $C$ is a precrossed complex we will denote by $C(x)$ the augmented complex

$$
\cdots \rightarrow C_{n}(x) \stackrel{d_{n}}{\longrightarrow} C_{n-1}(x) \rightarrow \cdots \rightarrow C_{3}(x) \stackrel{d_{3}}{\longrightarrow} C_{2}(x) \stackrel{d_{2}}{\longrightarrow} C_{1}(x) .
$$

If $u: x \rightarrow y$ is a morphism of $C_{\leqslant 1}$ and $n \geqslant 1$, we will call the map $C_{n}(x) \rightarrow C_{n}(y)$ the action of $u$ on $C_{n}(x)$. In particular, an element of $C_{1}(x)$ acts on $C_{n}(x)$ for all $n \geqslant 1$.

Definition 2.2. A precrossed complex $C$ is a crossed complex if for all $x$ in $C_{0}$ the following conditions are satisfied:

- For every $n \geqslant 3$, the group $C_{n}(x)$ is abelian;

- For every $u$ in $C_{2}(x)$, the element $d_{2}(u)$ of $C_{1}(x)$ acts

- By conjugation by $u$ on $C_{2}(x)$;

- Trivially on $C_{n}(x)$ for $n \geqslant 3$.

Definition 2.3. Let $C$ and $D$ be two crossed complexes. A morphism of crossed complexes $f: C \rightarrow D$ consists of

- A functor $f_{\leqslant 1}: C_{\leqslant 1} \rightarrow D_{\leqslant 1}$;

- A natural transformation $f_{\geqslant 2}: C_{\geqslant 2} \rightarrow D_{\geqslant 2} f_{\leqslant 1}$,

such that $f_{\geqslant 2}$ is compatible with the augmentation, i.e., the square

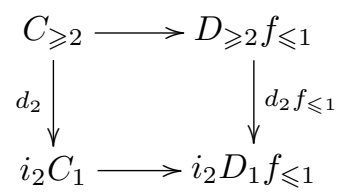

is commutative.

Explicitly, a morphism $f: C \rightarrow D$ is given by

- A functor $f_{\leqslant 1}: C_{\leqslant 1} \rightarrow D_{\leqslant 1}$;

- For every $n \geqslant 2$ and every $x$ in $C_{0}$, a group morphism $f_{n}: C_{n}(x) \rightarrow D_{n}\left(f_{0}(x)\right)$, where $f_{0}$ denotes the restriction of $f_{\leqslant 1}$ to objects,

such that for every $x$ in $C_{0}$, the diagram

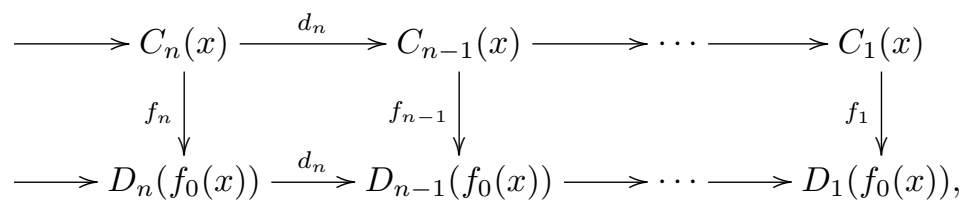

where $f_{1}$ denotes the restriction of $f_{\leqslant 1}$ to $C_{1}(x)$, is commutative. We will often simply write $f$ for $f_{n}$.

We will denote the category of crossed complexes by $\mathbf{C r C}$.

\subsection{The equivalence with strict $\infty$-groupoids}

Let $G$ be a strict $\infty$-groupoid. One can associate to $G$ a precrossed complex $C$ in the following way:

- The groupoid $C_{\leqslant 1}$ is the 1-truncation of $G$ obtained from $G$ by throwing out the $n$-arrows for $n \geqslant 2$; 
- $C_{n}(x)$ is the set of $n$-arrows $u$ of $G$ whose source is an iterated unit of an object, that is such that there exists an object $x$ of $G$ such that $s_{n-1}(u)=1_{x}$;

- The group law on $C_{n}(x)$ is induced by the composition $*_{0}^{n}: G_{n} \times G_{0} G_{n} \rightarrow G_{n}$;

- $d_{n}: C_{n}(x) \rightarrow C_{n-1}(x)$ is induced by the target map $t_{n-1}: G_{n} \rightarrow G_{n-1}$;

- For $u: x \rightarrow y$ a morphism in $C_{\leqslant 1}$ and $v$ a morphism in $C_{n}(x)$ for $n \geqslant 2$, the action of $u$ on $v$ is

$$
u *_{0} v *_{0} w_{0}(u) .
$$

This precrossed complex is a crossed complex (see paragraph 3 of [5]). Moreover, this construction defines a functor $\mathrm{A}: \infty$-Grp $\rightarrow$ CrC.

Theorem 2.4 (Brown-Higgins). The functor $\mathrm{A}: \infty-\mathbf{G r p} \rightarrow \mathbf{C r C}$ is an equivalence of categories.

Proof. See Theorem 4.1 of [5].

\subsection{The model structure on crossed complexes}

Definition 2.5. Let $C$ be a crossed complex. The set of connected components of $C$ is

$$
\pi_{0}(C)=\pi_{0}\left(C_{\leqslant 1}\right)
$$

For $x$ in $C_{0}$, the fundamental group of $C$ at $x$ is

$$
\pi_{1}(C, x)=\operatorname{Coker}\left(d_{2}: C_{2}(x) \rightarrow C_{1}(x)\right),
$$

and for $n \geqslant 2$, the $n$-th homotopy group of $C$ at $x$ is

$$
\pi_{n}(C, x)=H_{n}(C(x)) .
$$

It is clear that $\pi_{0}$ defines a functor from the category of crossed complexes to the category of sets and that for $n \geqslant 1, \pi_{n}$ defines a functor from the category of pointed crossed complexes to the category of groups.

Definition 2.6. A morphism $f: C \rightarrow D$ of crossed complexes is a weak equivalence of crossed complexes (see [4]) if the map $\pi_{0}(f): \pi_{0}(C) \rightarrow \pi_{0}(D)$ is a bijection and if for every $x$ in $C_{0}$ and every $n \geqslant 1$, the morphism $\pi_{n}(f, x): \pi_{n}(C, x) \rightarrow \pi_{n}(D, f(x))$ is an isomorphism.

Definition 2.7. Let $f: C \rightarrow D$ be a morphism of crossed complexes. The morphism $f$ is a trivial fibration of crossed complexes (see Proposition 2.2 (ii) of [4]) if the following conditions are satisfied:

- For every $y$ in $D_{0}$, there exists $x$ in $C_{0}$ such that $f(x)=y$;

- For every $x, y$ in $C_{0}$ and every morphism $v: f(x) \rightarrow f(y)$ in $D_{\leqslant 1}$, there exists a morphism $u: x \rightarrow y$ in $C_{\leqslant 1}$ such that $f(u)=v$;

- For every $n \geqslant 2, x$ in $C_{0}, t$ in $C_{n-1}(x)$ and every $v$ in $D_{n}(f(x))$ such that $d_{n}(v)=f(t)$, there exists $u$ in $C_{n}(x)$ such that $d_{n}(u)=t$ and $f(u)=v$.

Theorem 2.8 (Brown-Golasiński). The weak equivalences and trivial fibrations of crossed complexes define a model category structure on the category of crossed complexes.

Proof. See Theorem 2.12 of [4]. 


\subsection{The model structure on strict $\infty$-groupoids}

One obtains a model category structure on strict $\infty$-groupoids by transferring the model structure on crossed complexes defined in the previous section via the equivalence of categories $\mathrm{A}: \infty-\mathbf{G r p} \rightarrow \mathbf{C r C}$. We will call this model structure the Brown-Golasiński model structure. A morphism $f$ of strict $\infty$-groupoids is a weak equivalence (respectively a trivial fibration) for the Brown-Golasiński model structure if and only if $\mathrm{A}(f)$ is a weak equivalence (respectively a trivial fibration) of crossed complexes. We will denote these classes by $\mathcal{W}_{\mathrm{cc}}$ and $\mathcal{T} \mathcal{F}_{\mathrm{cc}}$ respectively.

We now describe these two classes more explicitly.

Proposition 2.9. We have $\mathcal{W}_{\mathrm{cc}}=\mathcal{W}_{\mathrm{gr}}$. In other words, a morphism $f: G \rightarrow H$ of strict $\infty$-groupoids is a weak equivalence of strict $\infty$-groupoids if and only if the morphism $\mathrm{A}(f): \mathrm{A}(G) \rightarrow \mathrm{A}(H)$ is a weak equivalence of crossed complexes.

Proof. Since the two notions of weak equivalences are defined in terms of homotopy groups, it suffices to show that the two notions of homotopy groups coincide.

Let $G$ be a groupoid. By definition, $\pi_{0}(G)=\pi_{0}(\mathrm{~A}(G))$.

Let $x$ be an object of $G$. By definition, $\pi_{1}(\mathrm{~A}(G), x)$ is the cokernel of the morphism $\mathrm{A}(G)_{2}(x) \rightarrow \mathrm{A}(G)_{1}(x)$. The set $\mathrm{A}(G)_{1}(x)$ is the set of 1-arrows $u: x \rightarrow x$ in $G$, and two such arrows $u, u^{\prime}$ are identified in the cokernel if and only if there exists a 2-arrow from $1_{x}$ to $u *_{0} u^{\prime-1}$ in $G$. This condition is equivalent to the existence of a 2-arrow from $u$ to $u^{\prime}$. Hence $\pi_{1}(G, x)=\pi_{1}(\mathrm{~A}(G), x)$.

Let $n \geqslant 2$. The kernel of the map $d_{n}: \mathrm{A}(G)_{n}(x) \rightarrow \mathrm{A}(G)_{n-1}(x)$ is the set $G_{n}(x, x)$. Thus the same argument as in dimension 1 shows that $\pi_{n}(G, x)=\pi_{n}(\mathrm{~A}(G), x)$.

Proposition 2.10. A morphism $f: G \rightarrow H$ of strict $\infty$-groupoids is in $\mathcal{T F}_{\mathrm{cc}}$ if and only if it satisfies the following conditions:

- For every object $y$ of $H$, there exists an object $x$ of $G$ such that $f(x)=y$;

- For every pair $(x, y)$ of objects of $G$, the map

$$
G(x, y)_{0} \rightarrow G(f(x), f(y))_{0}
$$

induced by $f$ is a surjection;

- For all $n \geqslant 2$, every object $x$ of $G$ and every $(n-1)$-arrow $u: 1_{x} \rightarrow 1_{x}$, the map

$$
G\left(1_{x}, u\right)_{0} \rightarrow H\left(1_{f(x)}, f(u)\right)_{0}
$$

is surjective.

Proof. By definition, $f$ is in $\mathcal{T F}_{\mathrm{cc}}$ if and only if $\mathrm{A}(f)$ is a trivial fibration of crossed complexes. This proposition is then just a matter of translation using the definition of the functor $\mathrm{A}$.

\section{The folk model structure on $\infty$-Grp}

This section shows that the folk model structure on $\infty$-Cat defined in [10] transfers to $\infty$-Grp via the inclusion functor

$$
U: \infty \text {-Grp } \rightarrow \infty \text {-Cat. }
$$

We first give a brief review of the main results of [10] and introduce the material needed to prove the transfer theorem. 


\subsection{The folk model structure on $\infty$-Cat}

Given an $\infty$-category $C$, we define reversible cells in $C$ and the relation of $\omega$-equivalence between cells of $C$ by mutual coinduction on $n \geqslant 0$.

Definition 3.1. Let $n \in \mathbb{N}$ :

- An $n$-cell $x$ is $\omega$-equivalent to an $n$-cell $y$ if there is a reversible $(n+1)$-cell $u: x \rightarrow y$;

- An $(n+1)$-cell $u: x \rightarrow y$ is reversible if there is an $(n+1)$-cell $\bar{u}: y \rightarrow x$ such that $\bar{u} *_{n} u$ is $\omega$-equivalent to $1_{x}$ and $u *_{n} \bar{u}$ is $\omega$-equivalent to $1_{y}$.

Note that, for each $r \geqslant 0$, if two cells are $r$-equivalent in the sense of [12], then they are $\omega$-equivalent, with the converse being false. We also refer to [7] for a gentle introduction to coinductive methods. Remark also that if $G$ is an $\infty$-groupoid, any $(n+1)$-cell $u$ of $G$ is reversible and the cell $\bar{u}$ whose existence is stated in Definition 3.1 is of course just $u^{-1}$.

Let $\mathcal{W}$ denote the class of those morphisms $f: C \rightarrow D$ satisfying the following two conditions:

1. For each 0-cell $y$ in $D$, there is a 0 -cell $x$ in $C$ such that $f x$ is $\omega$-equivalent to $y$;

2. For each pair $\left(x, x^{\prime}\right)$ of parallel $n$-cells in $C$, where $n \geqslant 0$ and each $(n+1)$-cell $v: f x \rightarrow f x^{\prime}$, there is an $(n+1)$-cell $u: x \rightarrow x^{\prime}$ such that $f u$ is $\omega$-equivalent to $v$.

Now for each $n \geqslant 0$, we define the $n$-globe $\mathbf{O}^{n}$ as the free $\infty$-category generated by the representable globular set $Y(n)=\mathbf{O}(n,-)$. Thus $Y(n)$ has exactly one $n$-cell, two $k$-cells for each $k<n$ and no $k$-cell for $k>n$. Let also $\partial Y(n)$ be the globular set having the same cells as $Y(n)$ except in dimension $n$ where $(\partial Y(n))_{n}=\emptyset$. We denote by $\partial \mathbf{O}^{n}$ the free $\infty$-category on $\partial Y(n)$. We finally have, for each $n \geqslant 0$, an inclusion morphism

$$
\mathbf{i}_{n}: \partial \mathbf{O}^{n} \rightarrow \mathbf{O}^{n}
$$

The set $\left\{\mathbf{i}_{n} \mid n \in \mathbb{N}\right\}$ is denoted by $I$.

A map is a trivial fibration if it has the right-lifting property with respect to $I$ and a cofibration if it has the left-lifting property with respect to all trivial fibrations.

Proposition 3.2. Any $\infty$-functor $f$ factors as $f=p \circ k$ where $p$ is a trivial fibration and $k$ is a cofibration.

Proof. By a standard application of the small object argument, using the fact that $\infty$-Cat is locally presentable.

On the other hand the maps $\sigma_{n}, \tau_{n}: n \rightarrow n+1$ in the globular category $\mathbf{O}$ (see Section 1) induce corresponding maps from $\mathbf{O}^{n}$ to $\mathbf{O}^{n+1}$, of the form $\mathbf{i}_{n+1} \circ \hat{\sigma}_{n}$ and 
$\mathbf{i}_{n+1} \circ \hat{\tau}_{n}$ respectively, where $\hat{\sigma}_{n}, \hat{\tau}_{n}: \mathbf{O}^{n} \rightarrow \partial \mathbf{O}^{n+1}$. Moreover, we get a pushout diagram

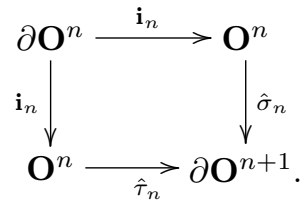

Now the above pushout determines a canonical map

$$
\mathbf{o}_{n}: \partial \mathbf{O}^{n+1} \rightarrow \mathbf{O}^{n}
$$

such that $\mathbf{o}_{n} \circ \hat{\sigma}_{n}=\mathbf{o}_{n} \circ \hat{\tau}_{n}=\mathrm{id}_{\mathbf{O}^{n}}$. Proposition 3.2 applies to $\mathbf{o}_{n}$, yielding an object $\mathbf{P}^{n}$ together with a trivial fibration $\mathbf{p}_{n}: \mathbf{P}^{n} \rightarrow \mathbf{O}^{n}$ and a cofibration $\mathbf{k}_{n}: \partial \mathbf{O}^{n+1} \rightarrow \mathbf{P}^{n}$ satisfying $\mathbf{o}_{n}=\mathbf{p}_{n} \circ \mathbf{k}_{n}$. We finally define $\mathbf{j}_{n}: \mathbf{O}^{n} \rightarrow \mathbf{P}^{n}$ as $\mathbf{k}_{n} \circ \hat{\sigma}_{n}$ and

$$
J=\left\{\mathbf{j}_{n} \mid n \in \mathbb{N}\right\} .
$$

Theorem 3.3. There is a cofibrantly generated model structure on $\infty$-Cat where $\mathcal{W}$ is the class of weak equivalences, $I$ a set of generating cofibrations and $J$ a set of generating trivial cofibrations.

This statement is in fact [10, Theorem 4.39] and the main result of that article.

\subsection{Path object}

Let $C$ be an object in a model category and $\Delta_{C}: C \rightarrow C \times C$ be the diagonal map. A path object for $C$ consists in an object $P_{C}$ together with a factorization of $\Delta_{C}$ of the form

$$
C \underset{\Delta_{C}}{\stackrel{j}{\longrightarrow} P_{C} \stackrel{p}{\longrightarrow}} C \times C,
$$

where $p$ is a fibration and $j$ is a weak equivalence. Such a $P_{C}$ is not unique: in the case of $\infty$-Cat, one particular choice is given by the functor $\Gamma$ we now describe. We first define, by induction on $n$, the notion of $n$-cylinder between $n$-cells $x, y$ of an $\infty$-category $C$. Some notation will be useful: for each $n$-cell $x$, we denote by $x^{b}$ its 0 -source $s_{0} x$ and by $x^{\sharp}$ its 0 -target $t_{0} x$. Now let $C$ be an $\infty$-category and $x, y$ two 0 -cells in it. There is an $\infty$-category $C(x, y)$ whose $n$-cells are the $(n+1)$-cells $u$ of $C$ such that $u^{b}=x$ and $u^{\sharp}=y$. Whenever $u$ is such an $(n+1)$-cell of $C$, we denote by $[u]$ the corresponding $n$-cell of $C(x, y)$. Finally, let $x, y, z$ be 0 -cells of $C$. Each 1-cell $u: x \rightarrow y$ determines an $\infty$-functor $-\cdot u: C(y, z) \rightarrow C(x, z)$ given by $[v]$. $u=\left[v *_{0} u\right]$. Likewise, $u: y \rightarrow z$ determines an $\infty$-functor $u \cdot-: C(x, y) \rightarrow C(x, z)$ by $u \cdot[v]=\left[u *_{0} v\right]$.

\section{Definition 3.4.}

1. A 0 -cylinder $U: x \curvearrowright y$ in $C$ is given by a reversible 1-cell $U^{\natural}: x \rightarrow y$;

2. If $n>0$, an $n$-cylinder $U: x \curvearrowright y$ in $C$ is given by two reversible 1-cells $U^{\mathrm{b}}: x^{\mathrm{b}} \rightarrow$ $y^{b}$ and $U^{\sharp}: x^{\sharp} \rightarrow y^{\sharp}$, together with some $(n-1)$-cylinder $[U]: U^{\sharp} \cdot[x] \curvearrowright[y] \cdot U^{b}$ in the $\infty$-category $\left[x^{b}, y^{\sharp}\right]=C\left(x^{b}, y^{\sharp}\right)$. 

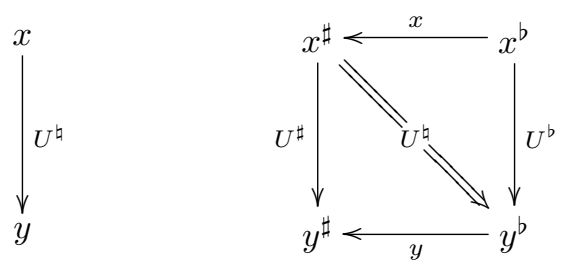

Figure 1: $n$-cylinders for $n=0,1$

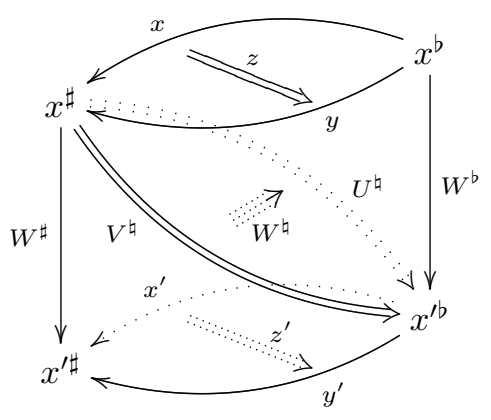

Figure 2: Source and target of a 2-cylinder

If $U: x \curvearrowright y$ is an $n$-cylinder in $C$, we write $\pi_{C}^{1} U$ and $\pi_{C}^{2} U$ for the $n$-cells $x$ and $y$, or simply $\pi^{1} U$ and $\pi^{2} U$. Figure 1 represents $n$-cylinders for $n=0$ and $n=1$. For each $n \in \mathbb{N}$, any $(n+1)$-cylinder $W: z \curvearrowright z^{\prime}$ in an $\infty$-category $C$ determines a pair of $n$-cylinders in $C$ :

Definition 3.5. The source $n$-cylinder $U: x \curvearrowright x^{\prime}$ and the target $n$-cylinder $V: y \curvearrowright$ $y^{\prime}$ of the $(n+1)$-cylinder $W: z \curvearrowright z^{\prime}$ between $(n+1)$-cells $z: x \rightarrow y$ and $z^{\prime}: x^{\prime} \rightarrow y^{\prime}$ are defined inductively by:

- If $n=0$, then $U^{\natural}=W^{b}$ and $V^{\natural}=W^{\sharp}$;

- If $n>0$, then $U^{\mathrm{b}}=V^{\mathrm{b}}=W^{\mathrm{b}}$ and $U^{\sharp}=V^{\sharp}=W^{\sharp}$, whereas the two $(n-1)$-cylinders $[U]$ and $[V]$ are respectively defined as the source and the target of the $n$-cylinder $[W]$ in the $\infty$-category $\left[z^{b}, z^{\prime \sharp}\right]$.

If $W$ has source $U$ and target $V$ we write $W: U \rightarrow V$ or $W: U \rightarrow V \mid z \curvearrowright z^{\prime}$ (see Figure 2). It turns out that the source and target maps so defined satisfy the globular relations, so that the correspondence

$$
n \mapsto\{U \mid U \text { is an } n \text {-cylinder in } C\}
$$

determines a globular set $\Gamma(C)$. We now turn to trivial $n$-cylinders:

Definition 3.6. The trivial $n$-cylinder $\tau x: x \curvearrowright x$ on the $n$-cell $x$ is defined inductively by:

- If $n=0$, then $(\tau x)^{\natural}=1_{x}$;

- If $n>0$, then $(\tau x)^{b}=1_{x^{b}}$ and $(\tau x)^{\sharp}=1_{x^{\sharp}}$, whereas $[\tau x]$ is the trivial cylinder $\tau[x]$ in $\left[x^{b}, x^{\sharp}\right]$. 
We write $\tau_{C} x$ in case we need to mention the ambient $\infty$-category $C$.

Let us finally recall from $[\mathbf{1 0}$, Appendix $\mathrm{A}]$ that $\Gamma(C)$ becomes a strict $\infty$-category when defining units and compositions as follows:

Definition 3.7. Let $U: x \curvearrowright y$ be an $n$-cylinder. We define the $(n+1)$-cylinder $1_{U}$ : $U \rightarrow U \mid 1_{x} \curvearrowright 1_{y}$ by induction on $n$ :

- If $n=0$, then $\left(1_{U}\right)^{b}=\left(1_{U}\right)^{\sharp}=U^{\natural}$, whereas $\left[1_{U}\right]=\tau\left[1_{U^{\natural}}\right]$;

- If $n>0$, then $\left(1_{U}\right)^{b}=U^{\mathrm{b}}$ and $\left(1_{U}\right)^{\sharp}=U^{\sharp}$, whereas $\left[1_{U}\right]=1_{[U]}$.

In order to define composition, we first introduce the operation of concatenation:

Definition 3.8. Let $U \mid x \curvearrowright y$ and $V \mid y \curvearrowright z$ be two $n$-cylinders. The concatenation $V * U \mid x \curvearrowright z$ of $U$ and $V$ is defined by induction on $n$ :

- If $n=0$, then $(V * U)^{\natural}=V^{\natural} *_{0} U^{\natural}$;

- If $n>0$, then $(V * U)^{b}=V^{b} *_{0} U^{b},(V * U)^{\sharp}=V^{\sharp} *_{0} U^{\sharp}$ and $[V * U]=[V] \cdot U^{b} *$ $V^{\sharp} \cdot[U]$.

Definition 3.9. Let $m \geqslant 1,0 \leqslant n<m$ and $U\left|x \curvearrowright x^{\prime}, V\right| y \curvearrowright y^{\prime}$ two $m$-cylinders such that $t_{n}(U)=s_{n}(V)$. The composition $V *_{n} U \mid y *_{n} x \curvearrowright y^{\prime} *_{n} x^{\prime}$ is defined by induction on $n$ as follows:

- $\left(V *_{0} U\right)^{\mathrm{b}}=U^{\mathrm{b}},\left(V *_{0} U\right)^{\sharp}=V^{\sharp}$ and $\left[V *_{0} U\right]=y^{\prime} \cdot[U] *[V] \cdot x$;

- If $n>0$, then $\left(V *_{n} U\right)^{\mathrm{b}}=U^{\mathrm{b}}=V^{\mathrm{b}},\left(U *_{n} V\right)^{\sharp}=U^{\sharp}=V^{\sharp}$ and $\left[V *_{n} U\right]=$ $[V] *_{n-1}[U]$.

Note that explicit formulas may be found in [11].

For example, Figure 3 below shows the composition $V *_{0} U$ of two 1-cylinders $U: x \curvearrowright x^{\prime}$ and $V: y \curvearrowright y^{\prime}$ such that $U^{\sharp}=V^{b}$. Precisely, the composite $V *_{0} U$ is the 1-cylinder $W: z \curvearrowright z^{\prime}$ where $z=y *_{0} x, z^{\prime}=y^{\prime} *_{0} x^{\prime}, W^{b}=U^{b}, W^{\sharp}=V^{\sharp}$, and the 0 -cylinder $[W]$ of $C\left(z^{b}, z^{\prime \sharp}\right)$ is the reversible 1-cell of $C\left(z^{b}, z^{\prime \sharp}\right)$ given by the following corresponding reversible 2 -cell of $C$ :

$$
W^{\natural}=\left(y^{\prime} *_{0} U^{\natural}\right) *_{1}\left(V^{\natural} *_{0} x\right) .
$$

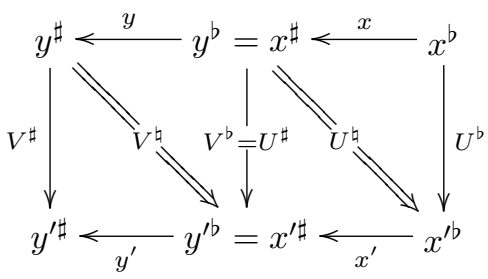

Figure 3: Composition of 1-cylinders

The following result summarizes the main properties of $\Gamma$ :

Theorem 3.10. The correspondence $C \mapsto \Gamma(C)$ induces an endofunctor on $\infty$-Cat, and $\pi^{1}, \pi^{2}: \Gamma \rightarrow \mathrm{id}, \tau: \mathrm{id} \rightarrow \Gamma$ are natural transformations. 
An additional property, of particular importance here, is that the functor $\Gamma$ preserves $\infty$-groupoids:

Lemma 3.11. If $G$ is an $\infty$-groupoid, so is $\Gamma(G)$.

Proof. We show, by induction on $n \geqslant 1$, that if $G$ is an $\infty$-groupoid and $W: U \rightarrow$ $V \mid x \curvearrowright y$ is an $n$-cylinder of $G$, there is an $n$-cylinder $W^{\prime}: V \rightarrow U \mid x^{-1} \curvearrowright y^{-1}$ such that $W^{\prime} *_{n-1} W=1_{U}$ and $W *_{n-1} W^{\prime}=1_{V}$.

Let $G$ be an $\infty$-groupoid and $W: U \rightarrow V \mid x \curvearrowright y$ a 1-cylinder of $G$. By definition, we get two 1-cells $U^{\natural}: x^{b} \rightarrow y^{b}, V^{\natural}: x^{\sharp} \rightarrow y^{\sharp}$ and a 2-cell $W^{\natural}: V^{\natural} *_{0} x \rightarrow y *_{0} U^{\natural}$ in $G$. Consider $W^{\natural-1}: y *_{0} U^{\natural} \rightarrow V^{\natural} *_{0} x$ the $*_{1}$-inverse of $W^{\natural}$ and build

$$
y^{-1} *_{0} W^{\natural-1} *_{0} x^{-1}: U^{\natural} *_{0} x^{-1} \rightarrow y^{-1} *_{0} V^{\natural} .
$$

If $W^{\prime}: V \rightarrow U \mid x^{-1} \curvearrowright y^{-1}$ is the 1-cylinder of $G$ defined by

$$
W^{\prime \natural}=y^{-1} *_{0} W^{\natural^{-1}} *_{0} x^{-1},
$$

we get

$$
W^{\prime} *_{0} W=1_{U} \quad \text { and } \quad W *_{0} W^{\prime}=1_{V},
$$

which proves the case $n=1$.

Let $n>1$ and suppose that the property holds for $n-1$. Let $G$ be an $\infty$-groupoid and $W: U \rightarrow V \mid x \curvearrowright y$ an $n$-cylinder of $G$. We get 1-cells $W^{b}: x^{b} \rightarrow y^{b}, W^{\sharp}: x^{\sharp} \rightarrow y^{\sharp}$ and an $(n-1)$-cylinder $[W]:[U] \rightarrow[V] \mid W^{\sharp} \cdot[x] \curvearrowright[y] \cdot W^{b}$ in $H=\left[x^{b}, y^{\sharp}\right]$. Now $H$ is an $\infty$-groupoid, so that the induction hypothesis applies and there is an $(n-1)$ cylinder in $H$

$$
[W]^{\prime}:[V] \rightarrow[U] \mid W^{\sharp} \cdot\left[x^{-1}\right] \curvearrowright\left[y^{-1}\right] \cdot W^{b}
$$

such that $[W]^{\prime} *_{n-2}[W]=1_{[U]}$ and $[W] *_{n-2}[W]^{\prime}=1_{[V]}$. Hence we may define an $n$-cylinder $W^{\prime}$ of $G$ by $W^{\prime b}=W^{b}, W^{\prime \sharp}=W^{\sharp}$ and $\left[W^{\prime}\right]=[W]^{\prime}$. By construction

$$
W^{\prime} *_{n-1} W=1_{U} \quad \text { and } \quad W *_{n-1} W^{\prime}=1_{V} .
$$

Remark 3.12. Let $n \geqslant 0$. The proof of the previous lemma actually shows that if $G$ is a strict $(\infty, n)$-category, then so is $\Gamma(G)$.

\subsection{Immersions}

We now introduce a class of morphisms which plays an important part in the proof of the transfer theorem.

Definition 3.13. An $\infty$-functor $f: C \rightarrow D$ belongs to the class $\mathcal{Z}$ of immersions if and only if there exist $\infty$-functors $g: D \rightarrow C$ and $h: D \rightarrow \Gamma(D)$ satisfying the following properties:

1. $g$ is a retraction of $f$, that is $g \circ f=\mathrm{id}_{C}$;

2. $\pi_{D}^{1} \circ h=f \circ g$ and $\pi_{D}^{2} \circ h=\operatorname{id}_{D}$;

3. $h \circ f=\tau_{D} \circ f$. In other words, $h$ is trivial on $f(C)$.

The following proposition summarizes the properties of immersions we need here. 

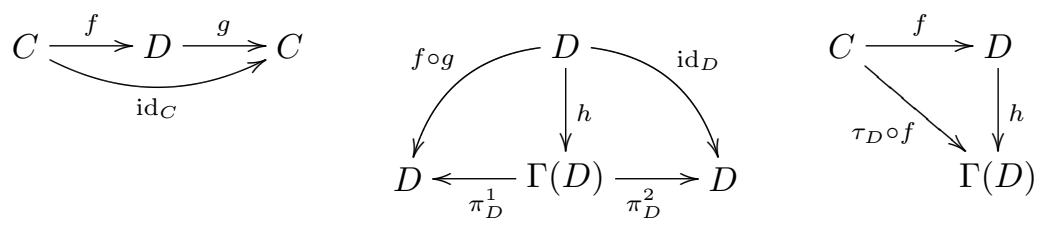

Figure 4: Immersions

Proposition 3.14. The class $\mathcal{Z}$ of immersions satisfies the following properties:

(i) $\mathcal{Z}$ is closed by pushout;

(ii) All trivial cofibrations are immersions;

(iii) All immersions are weak equivalences.

We refer to [10, Section 4.6] for the proofs of these statements.

\subsection{Transfer}

Let $\mathbf{C}, \mathbf{D}$ be two categories and $L: \mathbf{C} \rightarrow \mathbf{D}, R: \mathbf{D} \rightarrow \mathbf{C}$ be a pair of functors with $L$ left adjoint to $R$ and suppose that $\mathbf{C}$ is equipped with a model structure. We may define three classes of maps of $\mathbf{D}$ as follows:

- For each morphism $f$ of $\mathbf{D}, f \in \mathcal{W}_{\mathbf{D}}$ if and only if $R(f)$ is a weak equivalence in $\mathbf{C}$;

- For each morphism $f$ of $\mathbf{D}, f \in \mathcal{F}_{\mathbf{D}}$ if and only if $R(f)$ is a weak equivalence in C;

- $\mathcal{C}_{\mathbf{D}}$ is the class of maps having the left-lifting property with respect to $\mathcal{W}_{\mathbf{D}} \cap \mathcal{F}_{\mathbf{D}}$.

We say that $R$ creates a model structure on $\mathbf{D}$ if $\mathcal{W}_{\mathbf{D}}, \mathcal{F}_{\mathbf{D}}$ and $\mathcal{C}_{\mathbf{D}}$ are respectively the weak equivalences, fibrations and cofibrations of a model structure on $\mathbf{D}$. Sufficient conditions for this transfer to hold are given by [3, Proposition 2.3] or $[6$, Theorem 3.3]. The latter result immediately specializes to the following statement:

Proposition 3.15. Let $\mathbf{C}$ a cofibrantly generated model structure, with I a set of generating cofibrations and $J$ a set of generating trivial cofibrations. If $\mathbf{D}$ is locally presentable, then the following conditions are sufficient for $R$ to create a model structure on $\mathbf{D}$ :

(C1) The weak equivalences of $\mathbf{C}$ are closed under filtered colimits;

(C2) $R$ preserves filtered colimits;

(C3) For each generating trivial cofibration $j$ of $\mathbf{C}$, if $g$ is a pushout of $L(j)$ in $\mathbf{D}$, then $R(g)$ is a weak equivalence of $\mathbf{C}$.

Moreover, if these conditions hold, the model structure so defined is cofibrantly generated and has $L(I)$ as a set of generating cofibrations and $L(J)$ as a set of generating trivial cofibrations.

We now turn to the particular case where $\mathbf{C}=\infty$-Cat, $\mathbf{D}=\infty$-Grp and $R$ is the inclusion functor $U: \infty$-Grp $\rightarrow \infty$-Cat. Note that $U$ has a left-adjoint $F: \infty$-Cat $\rightarrow$ 
$\infty$-Grp building the free $\infty$-groupoid on an $\infty$-category, as well as a right-adjoint $M$, building to the maximal $\infty$-groupoid in an $\infty$-category. Let us first establish a few properties about the adjunction $F \dashv U$.

Let $T$ be the monad $U F$ on $\infty$-Cat. Remark that, for any $\infty$-groupoid $G$, the free $\infty$-groupoid on the underlying $\infty$-category $U(G)$ is naturally isomorphic to $G$ itself. In other words, the counit $\varepsilon: F U \rightarrow 1$ is a natural isomorphism. It follows that, for any $\infty$-groupoid $G$, we get an isomorphism

$$
\eta_{U(G)}: U(G) \rightarrow U F U(G),
$$

where $\eta$ denotes the unit of the adjunction. Now, for each $\infty$-category $C, T(C)$ is of the form $U(G)$ where $G$ is an $\infty$-groupoid and so is $\Gamma T(C)$ by Lemma 3.11, so that

$$
\eta_{\Gamma T(C)}: \Gamma T(C) \rightarrow T \Gamma T(C)
$$

is an isomorphism, as a special case of (1). Thus, we may define a natural transformation

$$
\lambda: T \Gamma \rightarrow \Gamma T
$$

by

$$
\lambda_{C}=\eta_{\Gamma T(C)}^{-1} \circ T \Gamma\left(\eta_{C}\right) .
$$

Note also that the monad multiplication $\mu: T^{2} \rightarrow T$ is also a natural isomorphism, and we get

$$
T\left(\eta_{C}\right)=\eta_{T(C)}=\mu_{C}^{-1} .
$$

We may now state the following result:

Lemma 3.16. The monad $T$ preserves immersions.

Proof. Let $f: C \rightarrow D$ be an immersion, and $f^{\prime}=T(f)$. By Definition 3.13, there are $g: D \rightarrow C$ and $h: D \rightarrow \Gamma(D)$ such that

$$
\begin{aligned}
g \circ f & =\operatorname{id}_{C}, \\
\pi_{D}^{1} \circ h & =f \circ g, \\
\pi_{D}^{2} \circ h & =\operatorname{id}_{D}, \\
h \circ f & =\tau_{D} \circ f .
\end{aligned}
$$

Let $g^{\prime}=T(g)$ and $h^{\prime}=\lambda_{D} \circ T(h)$. We need to establish the following equations:

$$
\begin{aligned}
g^{\prime} \circ f^{\prime} & =\operatorname{id}_{T(C)}, \\
\pi_{T(D)}^{1} \circ h^{\prime} & =f^{\prime} \circ g^{\prime}, \\
\pi_{T(D)}^{2} \circ h^{\prime} & =\operatorname{id}_{T(D)}, \\
h^{\prime} \circ f^{\prime} & =\tau_{T(D)} \circ f^{\prime} .
\end{aligned}
$$

Equation (3) is just functoriality. Let us prove (4). First remark that $\pi^{1}$ is a natural 
transformation, so that the following diagram commutes:

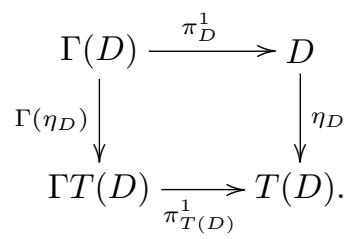

We may now build the following commutative diagram:

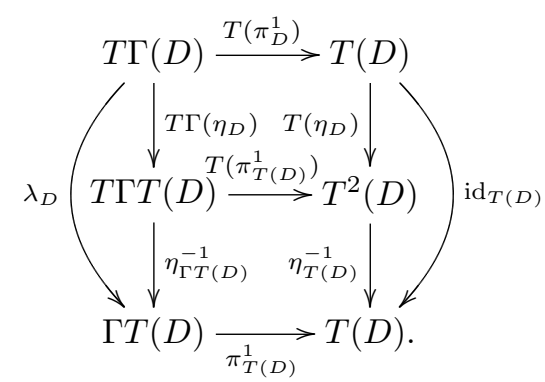

In fact the upper square is the image of (7) by $T$ and the lower square commutes by naturality of $\eta$. Hence

$$
\begin{aligned}
\pi_{T(D)}^{1} \circ h^{\prime} & =\pi_{T(D)}^{1} \circ \lambda_{D} \circ T(h) \\
& =T\left(\pi_{D}^{1}\right) \circ T(h) \\
& =T\left(\pi_{D}^{1} \circ h\right) \\
& =T(f \circ g) \\
& =f^{\prime} \circ g^{\prime},
\end{aligned}
$$

which gives (4). Likewise, we get the following commutative diagram:

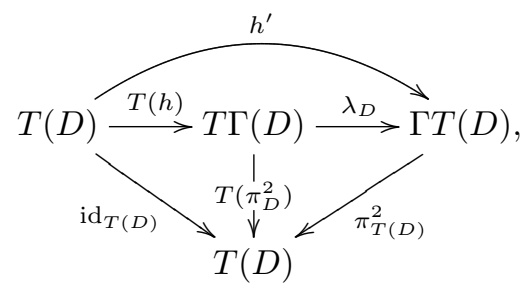

where the left-hand triangle commutes by applying $T$ to $(2)$, and the right-hand triangle commutes by replacing $\pi^{1}$ with $\pi^{2}$ in (8). Hence $\pi_{T(D)}^{2} \circ h^{\prime}=\mathrm{id}_{T(D)}$ and (5) is proved. Finally, by using the naturality of $\tau$ instead of $\pi^{1}$, we get a commutative 
diagram analogue to (8):

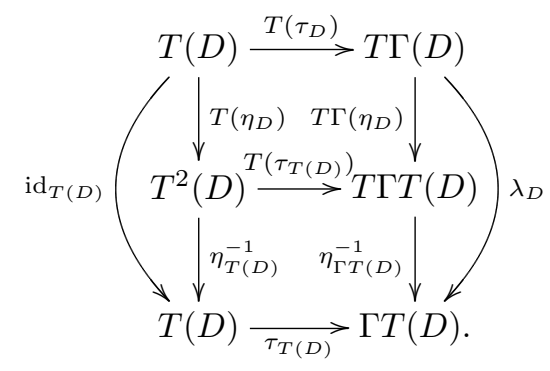

Hence

$$
\begin{aligned}
h^{\prime} \circ f^{\prime} & =\lambda_{D} \circ T(h) \circ T(f) \\
& =\lambda_{D} \circ T(h \circ f) \\
& =\lambda_{D} \circ T\left(\tau_{D} \circ f\right) \\
& =\lambda_{D} \circ T\left(\tau_{D}\right) \circ f^{\prime} \\
& =\tau_{T(D)} \circ f^{\prime}
\end{aligned}
$$

which gives (6) and ends the proof.

Lemma 3.17. Let $f: C \rightarrow D$ be an immersion and suppose that the following square is a pushout in $\infty$-Grp:

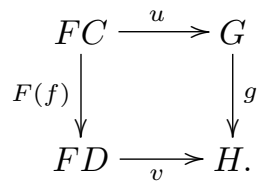

Then $U(g)$ is an immersion.

Proof. As $U$ is left adjoint to $M$, it preserves pushouts, so that the following square is a pushout in $\infty$-Cat:

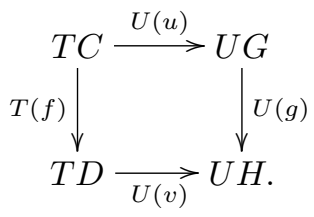

By Lemma 3.16, $T(f)$ is an immersion and so is its pushout $U(g)$, by Proposition 3.14(i).

Lemma 3.18. If $j$ is a generating trivial cofibration of $\infty$-Cat, and $g$ is a pushout of $F(j)$ in $\infty$-Grp, then $U(g)$ is a weak equivalence of $\infty$-Cat.

Proof. Let $j$ be a generating trivial cofibration of $\infty$-Cat, and $g$ be a pushout of $F(j)$ in $\infty$-Grp. By Proposition 3.14(ii), $j$ is an immersion and so is $U(g)$, by Lemma 3.17. By Proposition 3.14(iii), $U(g)$ is a weak equivalence.

We may finally state the main result of this section: 
Theorem 3.19. The forgetful functor $U: \infty-\mathbf{G r p} \rightarrow \infty$-Cat creates a model structure on $\infty$-Grp in which the weak equivalences are the morphisms $f$ such that $U(f)$ belongs to $\mathcal{W}$. Moreover, the model structure so defined has $\left(F\left(\mathbf{i}_{k}\right)\right)_{k \in \mathbb{N}}$ as a family of generating cofibrations and $\left(F\left(\mathbf{j}_{k}\right)\right)_{k \in \mathbb{N}}$ as a family of generating trivial cofibrations.

Proof. As the model structure on $\infty$-Cat is cofibrantly generated and $\infty$-Grp is locally presentable, Proposition 3.15 applies, and it suffices to check conditions (C1), $(\mathrm{C} 2)$ and $(\mathrm{C} 3)$. Condition $(\mathrm{C} 1)$ is proved in $[\mathbf{1 0}]$, and condition $(\mathrm{C} 2)$ follows from the fact that $U$ has a right-adjoint $M$, hence preserves colimits, and in particular filtered ones. Condition (C3) is Lemma 3.18. The statement about generating families follows from Proposition 3.15 .

Remark 3.20. Using Remark 3.12, one can easily adapt the proof of the previous theorem to show that a similar theorem holds for strict $(\infty, n)$-categories. In particular, the inclusion functor $(\infty, n)$-Cat $\rightarrow \infty$-Cat creates a model structure on $(\infty, n)$-Cat.

We call the model structure just defined the folk model structure on $\infty$-groupoids. We denote its weak equivalences by $\mathcal{W}_{\text {folk }}$ and its trivial fibrations by $\mathcal{T F}_{\text {folk }}$. Note that a morphism $f$ is in $\mathcal{T F}_{\text {folk }}$ if and only if $U(f)$ is a trivial fibration of $\infty$-Cat.

Proposition 3.21. A morphism $f: G \rightarrow H$ of $\infty$-groupoids belongs to $\mathcal{T F}_{\text {folk }}$ if and only if the following conditions are satisfied:

1. For every object $y$ of $H$, there exists an object $x$ of $G$ such that $f(x)=y$;

2. For all $n \geqslant 1$ and every pair $(u, v)$ of parallel $(n-1)$-arrows of $G$, the map

$$
G(u, v)_{0} \rightarrow H(f(u), f(v))_{0}
$$

is surjective.

Proof. By definition, $f$ belongs to $\mathcal{T F}_{\text {folk }}$ if and only if $U(f)$ has the right lifting property with respect to $I$. This proposition is then just a matter of translation.

\section{Comparison}

In this section, we show that the folk model structure on strict $\infty$-groupoids defined in the previous section coincides with the Brown-Golasiński model structure. To see this, it suffices to prove that they have the same weak equivalences and the same trivial fibrations.

Proposition 4.1. We have $\mathcal{W}_{\mathrm{gr}}=\mathcal{W}_{\text {folk }}=\mathcal{W}_{\mathrm{cc}}$

Proof. We first show that $\mathcal{W}_{\text {folk }}=\mathcal{W}_{\text {gr }}$. In a strict $\infty$-groupoid, two $n$-arrows $f$ and $g$ are $\omega$-equivalent if and only if there exists an $(n+1)$-arrow $a: f \rightarrow g$, that is if and only if $f$ and $g$ are homotopic. Therefore a morphism of strict $\infty$-groupoids is in $\mathcal{W}_{\text {folk }}$ if and only if it satisfies condition 4 of Proposition 1.7. The statement is thus exactly the equivalence between conditions 1 and 4 of this very proposition.

By Proposition 2.9, we have $\mathcal{W}_{\text {cc }}=\mathcal{W}_{\mathrm{gr}}$, hence the result.

Proposition 4.2. We have $\mathcal{T F}_{\text {folk }}=\mathcal{T F}_{\text {cc }}$. 
Proof. To prove the equivalence between the two notions of trivial fibrations, we will use the descriptions of these notions provided by Propositions 2.10 and 3.21. The conditions for being in $\mathcal{T F}_{\text {folk }}$ are a priori stronger. Let $f: G \rightarrow H$ be a in $\mathcal{T F}_{\text {cc }}$. Let us prove it is actually in $\mathcal{T F}_{\text {folk }}$. There is nothing to prove for the conditions in dimension 0 and 1 . Let $n \geqslant 2$ and let $u, v$ be two parallel $(n-1)$-arrows. We want to show that the map

$$
G(u, v)_{0} \rightarrow H(f(u), f(v))_{0}
$$

is surjective. Let $b$ be an $n$-arrow from $f(u)$ to $f(v)$ in $H$. Set $x=s_{0}(u)$. Then $b^{\prime}=1_{w_{0}(f(u))} *_{0} b$ is an $n$-arrow of $H$ from $1_{f(x)}$ to $w_{0}(f(u)) *_{0} f(v)$. Since the map

$$
G\left(1_{x}, w_{0}(u) *_{0} v\right)_{0} \rightarrow H\left(f\left(1_{x}\right), f\left(w_{0}(u) *_{0} v\right)\right)_{0}
$$

is surjective, there exists an $n$-arrow $a^{\prime}$ of $G$ from $1_{x}$ to $w_{0}(u) *_{0} v$ such that $f\left(a^{\prime}\right)=b^{\prime}$. Then, the $n$-arrow $a=1_{u} *_{0} a^{\prime}$ is from $u$ to $v$, and we have

$$
\begin{aligned}
f(a) & =f\left(1_{u} *_{0} a^{\prime}\right) \\
& =1_{f(u)} *_{0} b^{\prime} \\
& =1_{f(u)} *_{0} 1_{w_{0}(f(u))} *_{0} b \\
& =b .
\end{aligned}
$$

Theorem 4.3. The Brown-Golasiński model structure and the folk model structure on strict $\infty$-groupoids coincide.

Proof. By the two previous propositions, these model structures have the same weak equivalences and the same trivial fibrations.

\section{References}

[1] J. Adámek and J. Rosický, Locally presentable and accessible categories, London Math. Soc. Lecture Note Ser. 189, Cambridge University Press, Cambridge, 1994.

[2] D. Ara, Sur les $\infty$-groupoïdes de Grothendieck et une variante $\infty$-catégorique, Ph.D. thesis, Université Paris 7, 2010.

[3] T. Beke, Sheafifiable homotopy model categories. II. Journal of Pure and Applied Algebra 164 (2001), no. 3, 307-324.

[4] R. Brown and M. Golasiński, A model structure for the homotopy theory of crossed complexes, Cahiers Topologie et Géométrie Différentielle Catégoriques 30 (1989), no. 1, 61-82.

[5] R. Brown and P.J. Higgins, The equivalence of $\infty$-groupoids and crossed complexes, Cahiers Topologie Géom. Différentielle 22 (1981), no. 4, 371-386.

[6] S. Crans, Quillen closed model structures for sheaves, Journal of Pure and Applied Algebra 101 (1995), no. 1, 35-57.

[7] B. Jacobs and J. Rutten, A tutorial on (co)algebras and (co)induction. Bulletin of the EATCS 62 (1997), 222-259.

[8] A. Joyal and M. Tierney, Strong stacks and classifying spaces, in Category theory, Proc. Int. Conf. (Como/Italy 1990), Lecture Notes in Math. 1488 (1991), 213-236, Springer-Verlag, New York. 
[9] S. Lack, A Quillen model structure for 2-categories, K-Theory 26 (2002), no. 2, 171-205.

[10] Y. Lafont, F. Métayer, and K. Worytkiewicz, A folk model structure on omegacat, Advances in Mathematics 224 (2010), no. 3, 1183-1231.

[11] F. Métayer, Resolutions by polygraphs, Theory and Applications of Categories 11 (2003), no. 7, 148-184, http://www.tac.mta.ca/tac/.

[12] R. Street, The algebra of oriented simplexes, Journal of Pure and Applied Algebra 49 (1987), no. 3, 283-335.

Dimitri Ara ara@math.jussieu.fr

Institut Mathématiques de Jussieu, Université Paris Diderot - Paris 7, Case 7012, Bâtiment Chevaleret, 75205 Paris Cedex 13, France

François Métayer metayer@pps.jussieu.fr

Laboratoire PPS, Université Paris Diderot - Paris 7 \& CNRS, Case 7014, Bâtiment Chevaleret, 75205 Paris Cedex 13, France 